\title{
Alternative Measures to Chronic Pain Management
}

\author{
AK Mohiuddin", Treasurer \\ Dr. M. Nasirullah Memorial Trust, Tejgaon, Dhaka
}

DOI: $\underline{\text { 10.36348/SIJTCM.2019.v02i09.002 }}$

| Received: 13.09.2019 | Accepted: 21.09.2019 | Published: 30.11 .2019

*Corresponding author: AK Mohiuddin

"No pain no gain" still is one of the most widely used inspiring quote. With that spirit, chronic pain sufferers should find heaven on earth at some point of life. However, chronic pain is a widespread and complex set of conditions that are often difficult and expensive to treat. The Institute of Medicine (US) estimated that chronic pain affects over 100 million Americans and is associated with $\$ 874$ billion in healthcare costs annually. Of this, close to $10 \%$ is associated with the economic burden of prescription opioid overdose, abuse and dependency. Pain increases depression 3-5-fold and 50-80\% of chronic pain patients report insomnia that warrants clinical attention. Conventional pain killers are usually associated with a variety of adverse side effects, such as constipation, urinary retention, nausea, sedation, respiratory depression, low platelet count, sexual dysfunction and hyperalgesia.

Pain affects more than $65 \%$ cancer patients, distressing or intolerable in more than one-third of patients. Although, WHO described opioids as essential medicines for pain control but distribution shows substantial inequity, a less than $20 \%$ of the world's population consuming more than $90 \%$ of the world's supply. Famous celebrities like Bruce Lee, Chris Penn, Elvis Presley, Heath Ledger, Anna Nicole Smith died from opioid overdose. Also, some $85 \%$ of primary care physicians perceived their training in pain management to be inadequate in a Pan-European survey. Along with these, fear of dependence, prescription diversion, regulatory scrutiny, withdrawal symptoms, opioidrelated adverse events and deaths limit its use. Although, abuse and availability of medical cannabis are big issues, several studies support use of cannabis/marijuana in cancer pain management. Its social acceptability is gradually increasing around the world, but many studies oppose it's use or at least demand further investigation of benefit risk ratio. Longterm use of non-steroidal anti-inflammatory drugs (NSAIDs, like ibuprofen, naproxen) is often associated with many serious cardiovascular, gastrointestinal, renal, and other side effects [1].
Mild-to-moderate pain may be relieved by non-drug techniques alone. Moderate-to-severe pain may require medication in addition to nondrug techniques. Neuropathic pain estimates of $60 \%$ among those with chronic pain. Non-drug techniques can decrease pain intensity and can decrease awareness of pain to enable better coping some have been proven to work. Physical activity is the most basic, simple and well-known preventive and therapeutic measure for low back pain, particularly when the problem is caused by sedentary behavior. A study on arthritic patients revealed that walking 1000 more steps/day was associated with $16 \%$ to $18 \%$ less risk of developing functional limitation two years later [2]. Regular exercise can reduce physical impairments and improve participation in social, domestic, occupational, and recreational activities. Additional benefits of exercise include improvements in mobility, risk of falls, body weight, mental health, and metabolic abnormalities among arthritis patients. Approximately 70 to $85 \%$ of the western population will develop low back pain at least once during their lifetime. An 8-week moderate intensity aerobic exercise at $40 \%-60 \%$ of heart rate reserve combined with conventional physiotherapy, significantly reduced nearly $50 \%$ non-specific chronic low back pain (NSCLBP) [3].

Pain causes stress, and stress affects pain control chemicals in the brain, such as norepinephrine and serotonin. Behavioral approaches /relaxation training can help reduce muscle tension and stress, lower blood pressure, and control pain. Physical therapy should strongly be considered for the management of chronic pain to gradually increase flexibility and strength. Adding cognitive behavioral treatment (CBT) component to routine physical therapy reduces NSCLBP, disability and depression, fear of movement with enhanced of self-efficacy, enhancing functional capacity and QoL [4]. Companion of choice at birth increases the likelihood of vaginal births, reduces the need for caesarean sections, the use of forceps or vacuum during vaginal births, need to use pain medications during labor, shortens the duration of labor, improves women's satisfaction with care and improves Apgar scores [5]. Music therapy, in general, was 
effective in reducing pain intensity, fatigue, anxiety, and analgesic consumption in gynecological patients during the postoperative period.

Postoperative pain is not adequately managed in greater than $80 \%$ of patients in the US. Cryotherapy, should be widely used in the same because it is a noninvasive, very cost-effective, devoid of complication, and a very favorable patient report. $75 \%$ of patients with low back pain are treated with heating therapy and $7 \%$ with cooling therapy. Cooling therapy decreases tissue blood flow due to vasoconstriction, and that it also reduces tissue metabolism, oxygen utilization, and inflammation. Transcutaneous electrical nerve stimulation (TENS) as an adjunct is effective in reducing lower limb spasticity when applied for more than 30 minutes over nerve or muscle belly in chronic stroke survivors [6]. In France, work productivity loss contributed almost $90 \%$ of the total costs incurred by patients with fibromyalgia, with an economic cost of 13000 million euros annually which is around $\$ 100$ billion in US. Low FODMAP (fermentable oligo-, di-, mono-saccharides and polyols) and neurofeedback reduce pain intensity, improve attention and QoL more successfully than other psychological or multicomponent programs. Meditation-based interventions improve pain symptomology across a wide spectrum of pain-related disorders, including fibromyalgia, migraine, chronic pelvic pain [7].

Aromatherapy with orange oil can relieve pain in patients with fractured limbs. A study has shown that edible oil of orange can reduce breast pain caused by premenstrual syndrome. Pets provide companionship, unconditional love, and joy. An average 12 minutes exposure to a therapy dog reduces anxiety in $34 \%$ of fibromyalgia patients, together with reductions in pain and improvements in mood [8]. Pet therapy significantly increases patients' overall feeling of wellbeing and reduces anxiety about future uncertainty. There are three elements in hypnosis; absorption, dissociation, and suggestibility. Alleviation of pain with hypnosis, especially for patients who experienced obvious pain during the first-eye surgery also reported. Hypnosis session during subcutaneous venous port implantation under local anesthesia in cancer patients significantly improved patient satisfaction. Acupuncture and yoga found to be effective at reducing lymphoedema and pain in patients after breast cancer treatment $[9,10]$.

\section{REFERENCES}

1. Wong, R. S. (2019). Role of Nonsteroidal AntiInflammatory Drugs (NSAIDs) in Cancer Prevention and Cancer Promotion. Advances in pharmacological sciences, 2019.

2. Varma, V. R., Chuang, Y. F., Harris, G. C., Tan, E. J., \& Carlson, M. C. (2015). Low-intensity daily walking activity is associated with hippocampal volume in older adults. Hippocampus, 25(5), 605615.

3. Gatchel, R. J. (2018). Low Back Pain: Recent Advances and Perspectives. MDPI AGMultidisciplinary Digital Publishing Institute.

4. Hajihasani, A., Rouhani, M., Salavati, M., Hedayati, R., \& Kahlaee, A. H. (2018). The influence of cognitive behavioral therapy on pain, quality of life, and depression in patients receiving physical therapy for chronic low back pain: a systematic review. $P M \& R$. 11(2):167-176.

5. Kabakian-Khasholian, T., \& Portela, A. (2017). Companion of choice at birth: factors affecting implementation. BMC pregnancy and childbirth, 17(1), 265.

6. Mahmood, A., Veluswamy, S. K., Hombali, A., Mullick, A., Manikandan, N., \& Solomon, J. M. (2018). Effect of Transcutaneous Electrical Nerve Stimulation (TENS) on spasticity in adults with stroke: A systematic review and metaanalysis. Archives of physical medicine and rehabilitation, 100(4):751-768.

7. Mohiuddin, A. K. (2019). Non-drug pain management: opportunities to explore. Biomed Grid LLC, USA, 1-5.

8. Kline, J. A., Fisher, M. A., Pettit, K. L., Linville, C. T., \& Beck, A. M. (2019). Controlled clinical trial of canine therapy versus usual care to reduce patient anxiety in the emergency department. PloS one, 14(1), e0209232.

9. Chien, T. J., Liu, C. Y., \& Fang, C. J. (2019). The Effect of Acupuncture in Breast Cancer-Related Lymphoedema (BCRL): A Systematic Review and Meta-Analysis. Integrative cancer therapies, 18 , 1534735419866910.

10. Panchik, D., Masco, S., Zinnikas, P., Hillriegel, B., Lauder, T., Suttmann, E., ... \& Hermann, W. (2019). Effect of Exercise on Breast CancerRelated Lymphedema: What the Lymphatic Surgeon Needs to Know. Journal of reconstructive microsurgery, 35(01), 37-45. 